\title{
Eddy-Current Losses in the Solid Back-Iron of PM Machines for different Concentrated Fractional Pitch Windings
}

\author{
H. Polinder, M.J. Hoeijmakers, M. Scuotto \\ Electrical Power Processing \\ Delft University of Technology \\ Mekelweg 4, 2628 CD Delft \\ The Netherlands
}

\begin{abstract}
Permanent magnet (PM) machines with concentrated fractional-pitch stator windings are increasingly used because of their cost-effectiveness. In this paper, the eddycurrent losses in the solid back-iron of these machines are modeled. This model is applied to calculate the losses in the solid back-iron of the linear PM generator of the Archimedes Wave Swing (AWS) for different combinations of numbers of poles and numbers of teeth. In machines with fractional-pitch windings, these eddy-current losses are considerable and depend strongly on the combination of number of teeth and number of poles. In fractional pitch machines with a coil around very second tooth, these losses are excessive.
\end{abstract}

Index Terms-eddy currents, permanent-magnet machines, back-iron, fractional-pitch windings, concentrated windings, distributed windings

\section{INTRODUCTION}

Permanent magnet (PM) machines with concentrated fractional-pitch stator windings are increasingly used because of their cost-effectiveness. The objective of this paper is to model the eddy-current losses in the solid back-iron of these machines and to apply this model to calculate the losses in the solid back-iron of the linear PM generator of the Archimedes Wave Swing for different combinations of numbers of poles and numbers of teeth.

The Archimedes Wave Swing (AWS) is a system that converts ocean wave energy into electrical energy. It consists of an air-filled chamber, the bottom part of which is fixed to the sea bed, while the lid, called the floater, can move up and down under influence of the waves [1-3]. A linear PM generator is used to convert the mechanical power into electrical power.

Currently, a PM machine with distributed full-pitch stator windings is applied. It has one slot per pole per phase as illustrated in Fig. 1. The winding is a two-layer winding,

This work was supported in part by a Marie Curie Early Stage Research Training Fellowship of the European Community's Sixth Framework Programme under contract number MRTN-CT-2004-505166, the WAVETRAIN program. which makes it rather expensive.

PM generators with concentrated fractional pitch windings (as illustrated in Fig. 2 to 6) may be much cheaper because they have simple windings around one tooth that can be wound automatically. However, the magnetic field of these windings has more space harmonics, including sub-harmonics (harmonics with a wave length larger than twice the pole pitch). This leads to additional eddy-current losses in the magnets and the back-iron, which is made of solid steel to make a cheap and strong construction. If these losses are high, the magnets may become so hot that they demagnetize.

Different combinations of numbers of poles and numbers of teeth are also possible [4-8]. It is also possible to use windings around every second tooth. According to [9], the combination of 3 teeth with 3 coils per 4 poles gives unacceptably high losses, while these losses are negligible for full pitch windings. The contribution of this paper that it investigates other combinations of numbers of poles and numbers of teeth.

The paper starts with a short description of the AWS generator system. Next, the modeling of the space harmonics is described. Subsequently, the method of calculating the eddy-current losses is described and the results of the calculations are given. Then, the model is validated with measurements. Finally, conclusions are drawn.

\section{GENERATOR DESCRIPTION}

This section gives a very short description of the generator for the Archimedes Wave Swing. A more elaborate description is given in [1-3].

The requirements of the generator are:

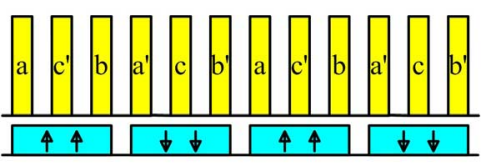

Fig. 1: Full pitch winding (I). 
- Maximum force (varying sinusoidally): $1 \mathrm{MN}$.

- Maximum speed (varying sinusoidally): $2.2 \mathrm{~m} / \mathrm{s}$.

If the maximum force is $1 \mathrm{MN}$ and the force varies sinusoidally as a function of time, the maximum RMS value of the force is $700 \mathrm{kN}$. This RMS value is important, because it is the most important factor influencing the losses and therefore the heating of the machine. In reality, the waves are irregular, so the RMS value of the force will be lower. In this paper, an RMS value of the force of $700 \mathrm{kN}$ is used.

The generator has an air-gap surface area of about $20 \mathrm{~m}^{2}$. Therefore, the RMS value for the force density (the force per square meter of air-gap surface area) is about $35 \mathrm{kN} / \mathrm{m}^{2}$.

The air gap and the magnets are dimensioned in such a way that the fundamental of the magnetic flux density due to the magnets in the air gap has an amplitude of about $1 \mathrm{~T}$. This is realistic with $\mathrm{NdFeB}$ magnets.

In order to obtain an RMS value of the force density of 35 $\mathrm{kN} / \mathrm{m}^{2}$, the amplitude of the current loading has to exceed 70 $\mathrm{kA} / \mathrm{m}$. In the machine with full pitch winding, a current per slot with an amplitude of $2.75 \mathrm{kA}$ results in a current loading of $82.5 \mathrm{kA} / \mathrm{m}$. This current is reasonable for a slot with a slot pitch of $33 \mathrm{~mm}$, a slot width of $16.5 \mathrm{~mm}$ and a slot height 80 $\mathrm{mm}$. The machines with fractional pitch windings all have the same stator laminations. Here, a current per slot with an amplitude of $5.5 \mathrm{kA}$ is used. This is reasonable for a slot with a slot pitch of $67 \mathrm{~mm}$, a slot width of $33 \mathrm{~mm}$ and a slot height $80 \mathrm{~mm}$. The resulting current loading is different for different combinations of numbers of teeth and numbers of poles because of the different winding factors [4]. However, this current should be high enough to generate the required force.

The voltage induced by the magnets (the back-EMF) is assumed to be sinusoidal. Also the currents are assumed to be sinusoidal. The three phase currents are given by

$i_{s a}=\hat{i}_{s} \cos (\omega t)$

$i_{s b}=\hat{i}_{s} \cos \left(\omega t-\frac{2}{3} \pi\right)$

$i_{s c}=\hat{i}_{s} \cos \left(\omega t-\frac{4}{3} \pi\right)$

The phase order ( $a b c$ or $a c b$ ) depends on the way the windings are distributed. When the phase order is $a c b$, the angular frequency $\omega$ is negative.

\section{MODELING WINDING HARMONICS}

\section{A. Modeling space harmonics of the windings}

This paper discusses the winding types mentioned in Table I. These winding types are used, because they have reasonable winding factors, as explained in [4].

The space harmonics of the magnetic flux density for the different windings are calculated in the following way.

First, the flux density in the air gap is determined for the case that only phase $a$ is conducting a current $\hat{l}_{s a}$. It is assumed that the stator teeth and slots can replaced by a smooth surface, placed at a distance $g_{\text {eff }}$ from the back-iron. This effective air gap $g_{\text {eff }}$ is the air gap multiplied by the Carter factor [10]. It is further assumed that the magnetic flux crosses the air gap perpendicularly and that the flux density is constant over a tooth pitch.

Next, this flux density is written as a Fourier series. The stator coordinate $x_{s}=0$ is chosen in such a way that only cosinusoidal components are present:

$B_{s a}\left(x_{s}\right)=\sum_{k=1}^{\infty} \hat{B}_{s k} \cos \left(k \frac{2 \pi x_{s}}{\lambda_{1}}\right)$

where

$x_{s}$ is the stator coordinate in the air gap, and

$\lambda_{1}$ is the wave length of the fundamental space harmonic.

The amplitudes of the space harmonics this flux density are calculated using the Fourier transform:

$\hat{B}_{s k}=\frac{2}{\lambda_{1}} \int_{0}^{\lambda_{1}} B_{s a}\left(x_{s}\right) \cos \left(k \frac{2 \pi x_{s}}{\lambda_{1}}\right) d x_{s}$

The flux density distributions of the other phases are equal to the flux density distribution of phase $a$ except for a shift in the position. If the currents are also written as a function of time as in (1), these flux density distributions can be written as $B_{s a}=\sum_{k=1}^{\infty} \hat{B}_{s k} \cos \left(k \frac{2 \pi x_{s}}{\lambda_{1}}\right) \cos (\omega t)$

$B_{s b}=\sum_{k=1}^{\infty} \hat{B}_{s k} \cos \left(k\left(\frac{2 \pi x_{s}}{\lambda_{1}}-\frac{2}{3} \pi\right)\right) \cos \left(\omega t-\frac{2}{3} \pi\right)$

$B_{s c}=\sum_{k=1}^{\infty} \hat{B}_{s k} \cos \left(k\left(\frac{2 \pi x_{s}}{\lambda_{1}}-\frac{4}{3} \pi\right)\right) \cos \left(\omega t-\frac{4}{3} \pi\right)$

To obtain the total flux density in the air gap, the three flux density distributions are added. After rewriting, this results in

$B_{s}=\sum_{k=1}^{\infty} B_{s k}$

where

$B_{s k}=\frac{3}{2} \hat{B}_{s k} \cos \left(\frac{2 k \pi}{\lambda_{1}} x_{s}-\omega t\right) \quad$ for $\quad k=1,4,7, \ldots$

$B_{s k}=\frac{3}{2} \hat{B}_{s k} \cos \left(\frac{2 k \pi}{\lambda_{1}} x_{s}+\omega t\right) \quad$ for $\quad k=2,5,8, \ldots$

$B_{s k}=0 \quad$ for $\quad k=3,6,9, \ldots$

TABLE I

WINDING TYPES CONSIDERED

\begin{tabular}{|l|l|}
\hline I & Full pitch with 1 slot per pole per phase \\
\hline II & Fractional pitch with 3 teeth with 3 coils per 2 poles \\
\hline III & Fractional pitch with 3 teeth with 3 coils per 4 poles \\
\hline IV & Fractional pitch with 9 teeth with 9 coils per 8 poles \\
\hline V & Fractional pitch with 9 teeth with 9 coils per 10 poles \\
\hline VI & Fractional pitch with 12 teeth with 12 coils per 10 poles \\
\hline VII & Fractional pitch with 12 teeth with 12 coils per 14 poles \\
\hline VIII & Fractional pitch with 6 teeth with 3 coils per 4 poles \\
\hline IX & Fractional pitch with 6 teeth with 3 coils per 8 poles \\
\hline $\mathrm{X}$ & Fractional pitch with 12 teeth with 6 coils per 10 poles \\
\hline XI & Fractional pitch with 12 teeth with 6 coils per 14 poles \\
\hline
\end{tabular}


The flux density with respect to the translator can then be calculated by writing the stator coordinate as a function of the translator coordinate:

$x_{s}=x_{r}+v t=x_{r} \pm \frac{\omega \tau_{p}}{\pi} t=x_{r} \pm \frac{\omega \lambda_{1}}{2 p_{\lambda} \pi} t$

where

$v$ is the speed of the translator;

$\tau_{p}$ is the pole pitch (the distance between two translator poles), $p_{\lambda}$ is the number of pole pairs within the wave length $\lambda_{1}$,

\pm is a + if the frequency $\omega$ is positive, and a - if the frequency $\omega$ is negative.

In the next subsections, the amplitudes of the space harmonics of the different winding types presented in Table I will be calculated. Table II gives the resulting amplitudes of the space harmonics of the magnetic flux density. Table III gives the speeds of these space harmonics with respect to the translator.

\section{B. Full pitch windings}

The generator with full-pitch winding (illustrated in Fig. 1) has a pole pitch of $\tau_{p}=0.1 \mathrm{~m}$ and a wave length of $\lambda_{1}=0.2 \mathrm{~m}$.

The flux density due to a current $\hat{l}_{s a}$ in phase $a$ of the full pitch winding of figure 1 can be can be calculated from Ampere's law as

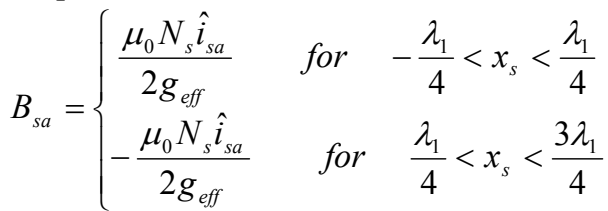

where

$\mu_{0}$ is the magnetic permeability in vacuum;

$N_{s}$ is the number of turns of a phase in a slot;

$g_{\text {eff }}$ is the effective air gap (including the Carter factor).

The amplitudes of the space harmonics can be calculated as $\hat{B}_{s k}=\frac{2}{k \pi} \frac{\mu_{0} N_{s} \hat{i}_{s a}}{g_{\text {eff }}} \sin \left(\frac{k \pi}{2}\right)$

\section{Fractional pitch windings with 3 teeth with 3 coils}

The generator with fractional pitch windings with 3 teeth with 3 coils as illustrated in Fig. 2 has a wave length of $\lambda_{1}=$ $0.2 \mathrm{~m}$. The generator with 2 poles per 3 teeth has a pole pitch of $\tau_{p}=0.1 \mathrm{~m}$ and the generator with 4 poles per 3 teeth has a pole pitch of $\tau_{p}=0.05 \mathrm{~m}$.

If the current in phase $a$ is $\hat{i}_{s a}$ and the currents in the other two phases are zero, the flux density can be calculated using Ampere's law as

$$
B_{s a}=\left\{\begin{array}{ccc}
\frac{2 \mu_{0} N_{s} \hat{i}_{s a}}{3 g_{e f f}} & \text { for } & -\frac{\lambda_{1}}{6}<x_{s}<\frac{\lambda_{1}}{6} \\
-\frac{\mu_{0} N_{s} \hat{i}_{s a}}{3 g_{\text {eff }}} & \text { for } & \frac{\lambda_{1}}{6}<x_{s}<\frac{5 \lambda_{1}}{6}
\end{array}\right.
$$

The amplitudes of the space harmonics of this flux density can be calculated as

$\hat{B}_{s k}=\frac{2}{k \pi} \sin \left(\frac{k \pi}{3}\right) \frac{N_{s} \hat{i}_{s a} \mu_{0}}{g_{\text {eff }}}$

\section{Fractional pitch windings with 9 teeth with 9 coils}

The generator with fractional pitch windings with 9 teeth with 9 coils as illustrated in Fig. 3 has a wave length of $\lambda_{1}=$ $0.6 \mathrm{~m}$. The generator with 8 poles per 9 teeth has a pole pitch of $\tau_{p}=0.075 \mathrm{~m}$ and the generator with 10 poles per 9 teeth has a pole pitch of $\tau_{p}=0.06 \mathrm{~m}$.

If the current in phase $a$ is $\hat{\imath}_{s a}$, and the currents in the other two phases are zero, the flux density can be calculated using Ampere's law as

$$
B_{s a}=\left\{\begin{aligned}
\frac{10 \mu_{0} N_{s} i_{s a}}{9 g_{\text {eff }}} & \text { for }-\frac{\lambda_{1}}{18}<x_{s}<\frac{\lambda_{1}}{18} \\
-\frac{8 \mu_{0} N_{s} i_{s a}}{9 g_{\text {eff }}} & \text { for }-\frac{\lambda_{1}}{6}<x_{s}<-\frac{\lambda_{1}}{18} ; \frac{\lambda_{1}}{18}<x_{s}<\frac{\lambda_{1}}{6} \\
\frac{\mu_{0} N_{s} i_{s a}}{9 g_{\text {eff }}} & \text { for } \frac{\lambda_{1}}{6}<x_{s}<\frac{5 \lambda_{1}}{6}
\end{aligned}\right.
$$

The amplitudes of the space harmonics can be calculated as

$\hat{B}_{s k}=\frac{2}{k \pi} \frac{N_{s} i_{s a} \mu_{0}}{g_{\text {eff }}}\left(2 \sin \left(\frac{k \pi}{9}\right)-\sin \left(\frac{k \pi}{3}\right)\right)$
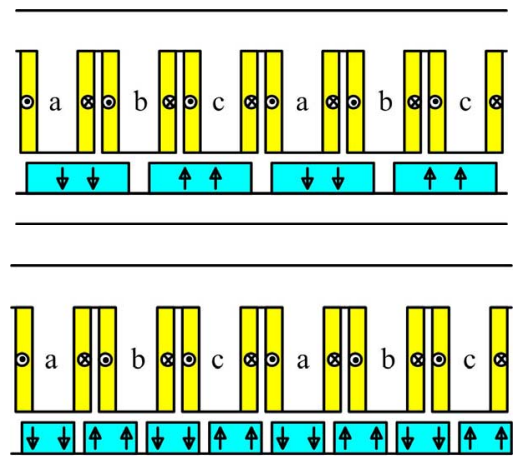

Fig. 2: Fractional pitch windings and 2 poles per 3 teeth with 3 coils (II) and with 4 poles per 3 teeth with 3 coils (III).
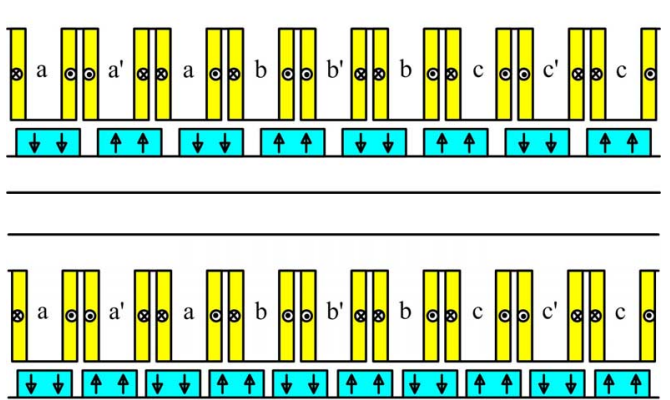

Fig. 3: Fractional pitch windings with 8 poles per 9 teeth with 9 coils (IV) and with 10 poles per 9 teeth with 9 coils (V). 


\section{E. Fractional pitch windings with 12 teeth with 12 coils}

The generator with fractional pitch windings with 12 teeth with 12 coils as illustrated in Fig. 4 has a wave length of $\lambda_{1}=$ $0.8 \mathrm{~m}$. The generator with 10 poles per 12 teeth has a pole pitch of $\tau_{p}=0.08 \mathrm{~m}$ and the generator with 14 poles per 12 teeth has a pole pitch of $\tau_{p}=0.0571 \mathrm{~m}$.

If the current in phase $a$ is $\hat{\imath}_{s a}$, and the currents in the other two phases are zero, the flux density in the air gap can be calculated using Ampere's law as

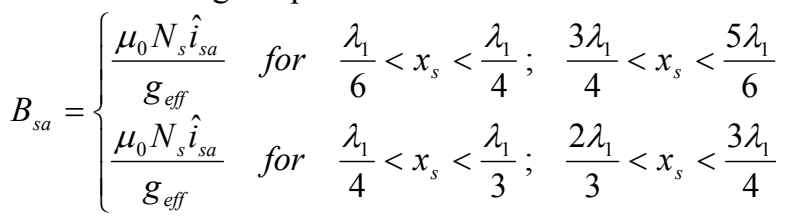

The amplitudes of the space harmonics can be calculated as

$\hat{B}_{s}=\frac{2 \mu_{0} N_{s} \hat{i}_{s a}}{k \pi g_{\text {eff }}}\left(2 \sin \left(\frac{k \pi}{2}\right)-\sin \left(\frac{k \pi}{3}\right)-\sin \left(\frac{2 k \pi}{3}\right)\right)$

\section{F. Fractional pitch windings: 6 teeth with 3 coils}

The generator with fractional pitch windings with 6 teeth with 3 coils as illustrated in Fig. 5 has a wave length of $\lambda_{1}=$ $0.4 \mathrm{~m}$. The generator with 4 poles per 6 teeth has a pole pitch of $\tau_{p}=0.1 \mathrm{~m}$ and the generator with 8 poles per 6 teeth has a pole pitch of $\tau_{p}=0.05 \mathrm{~m}$.

If the current in phase a is $\hat{\imath}_{s a}$, and the currents in the other two phases are zero, the flux density can be calculated using Ampere's law as

$B_{s a}= \begin{cases}\frac{5 \mu_{0} N_{s} \hat{i}_{s a}}{6 g_{\text {eff }}} & \text { for } \quad-\frac{\lambda_{1}}{12}<x_{s}<\frac{\lambda_{1}}{12} \\ -\frac{\mu_{0} N_{s} \hat{i}_{s a}}{6 g_{\text {eff }}} & \text { for } \quad \frac{\lambda_{1}}{12}<x_{s}<\frac{11 \lambda_{1}}{12}\end{cases}$

The amplitudes of the space harmonics can be calculated as $\hat{B}_{s k}=\frac{4 \mu_{0} N_{s} \hat{i}_{s a}}{k \pi g_{\text {eff }}} \sin \left(\frac{k \pi}{6}\right)$

\section{G. Fractional pitch windings: 12 teeth with 6 coils}

The generator with fractional pitch windings with 12 teeth with 6 coils as illustrated in Fig. 5 has a wave length of $\lambda_{1}=$ $0.8 \mathrm{~m}$. The generator with 10 poles per 12 teeth has a pole pitch of $\tau_{p}=0.08 \mathrm{~m}$ and the generator with 14 poles per 12 teeth has a pole pitch of $\tau_{p}=0.0571 \mathrm{~m}$.

If the current in phase $a$ is $\hat{\imath}_{s a}$, and the currents in the other two phases are zero, the flux density can be calculated using Ampere's law as

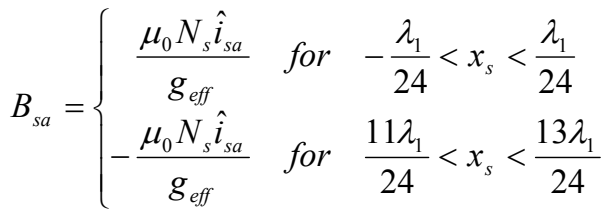

The amplitudes of the space harmonics can be calculated as

$$
\hat{B}_{s k}=\frac{2 \mu_{0} N_{s} \hat{i}_{s a}}{k \pi g_{\text {eff }}}\left(\sin \left(\frac{k \pi}{12}\right)+\sin \left(\frac{11 k \pi}{12}\right)\right)
$$
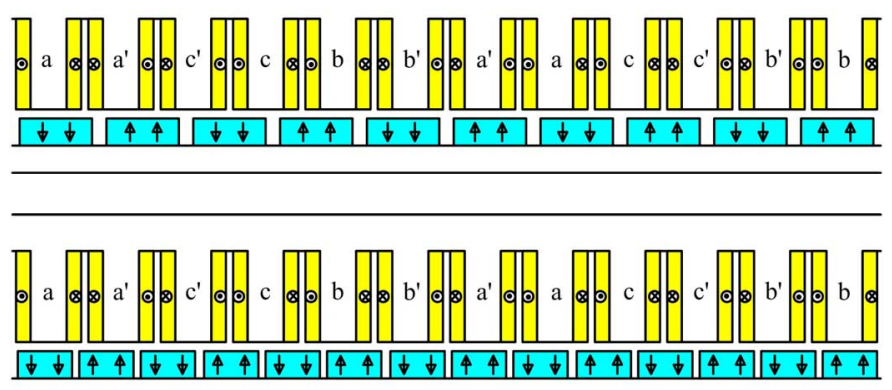

Fig. 4: Fractional pitch windings with 10 poles per 12 teeth with 12 coils (VI) and with 14 poles per 12 teeth with 12 coils (VII).
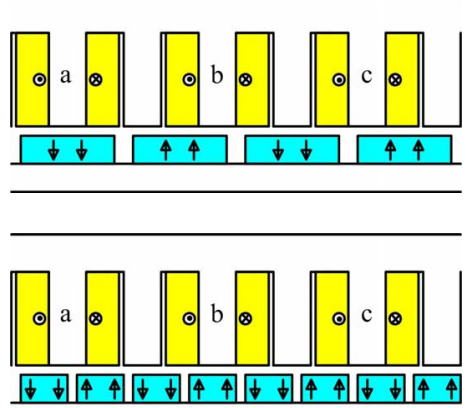

Fig. 5: Fractional pitch windings with 4 poles per 6 teeth with 3 coils (VIII) and with 8 poles per 6 teeth with 3 coils (IX)
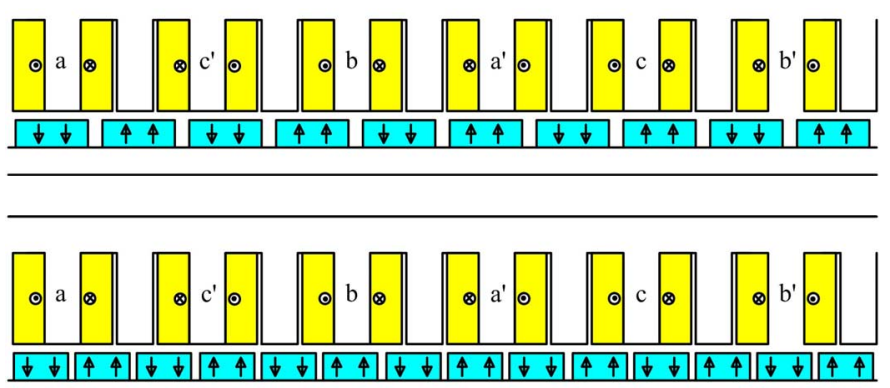

Fig. 6: Fractional pitch windings with 10 poles per 12 teeth with 6 coils (X) and with 14 poles per 12 teeth with 6 coils (XI).

TABLE II

AMPLITUDES OF THE SPACE HARMONICS OF THE FLUX DENSITY (MT)

\begin{tabular}{|r|r|r|r|r|r|r|r|r|}
\hline & 1 & 2 & 4 & 5 & 7 & 8 & 10 & 11 \\
\hline $\mathrm{I}$ & 160 & 0 & 0 & 32 & 23 & 0 & 0 & 15 \\
\hline $\mathrm{II}$ & 139 & 69 & -35 & -28 & 20 & 17 & -14 & -13 \\
\hline $\mathrm{III}$ & 139 & 69 & -35 & -28 & 20 & 17 & -14 & -13 \\
\hline $\mathrm{IV}$ & -29 & 34 & 113 & 91 & 10 & -4 & 3 & -1 \\
\hline $\mathrm{V}$ & -29 & 34 & 113 & 91 & 10 & -4 & 3 & -1 \\
\hline $\mathrm{VI}$ & 43 & 0 & 0 & 119 & -85 & 0 & 0 & -4 \\
\hline $\mathrm{VII}$ & 43 & 0 & 0 & 119 & -85 & 0 & 0 & -4 \\
\hline $\mathrm{VIII}$ & 320 & 277 & 139 & 64 & -46 & -69 & -55 & -29 \\
\hline $\mathrm{IX}$ & 320 & 277 & 139 & 64 & -46 & -69 & -55 & -29 \\
\hline $\mathrm{X}$ & 166 & 0 & 0 & 124 & 88 & 0 & 0 & 15 \\
\hline $\mathrm{XI}$ & 166 & 0 & 0 & 124 & 88 & 0 & 0 & 15 \\
\hline
\end{tabular}


TABLE III

SPEEDS OF THE SPACE HARMONICS OF THE FLUX DENSITY WITH REPSECT TO THE TRANSLATOR $(\mathrm{M} / \mathrm{S})$

\begin{tabular}{|r|r|r|r|r|r|r|r|r|}
\hline & 1 & 2 & 4 & 5 & 7 & 8 & 10 & 11 \\
\hline $\mathrm{I}$ & 0 & - & - & -2.64 & -1.89 & - & - & -2.40 \\
\hline $\mathrm{II}$ & 0 & -3.30 & -1.65 & -2.64 & -1.89 & -2.48 & -1.98 & -2.40 \\
\hline $\mathrm{III}$ & -6.60 & 0 & -3.30 & -1.32 & -2.83 & -1.65 & -2.64 & -1.80 \\
\hline $\mathrm{IV}$ & 6.60 & -6.60 & 0 & -3.96 & -0.94 & -3.30 & -1.32 & -3.00 \\
\hline $\mathrm{V}$ & -13.2 & 3.30 & -4.95 & 0 & -3.77 & -0.83 & -3.30 & -1.20 \\
\hline $\mathrm{VI}$ & -13.2 & 3.30 & -4.95 & 0 & -3.77 & -0.83 & -3.30 & -1.20 \\
\hline $\mathrm{VII}$ & 13.2 & -9.90 & 1.65 & -5.28 & 0 & -4.13 & -0.66 & -3.60 \\
\hline $\mathrm{VIII}$ & -6.60 & 0 & -3.30 & -1.32 & -2.83 & -1.65 & -2.64 & -1.80 \\
\hline $\mathrm{IX}$ & 6.60 & -6.60 & 0 & -3.96 & -0.94 & -3.30 & -1.32 & -3.00 \\
\hline $\mathrm{X}$ & -13.2 & 3.30 & -4.95 & 0 & -3.77 & -0.83 & -3.30 & -1.20 \\
\hline $\mathrm{XI}$ & 13.2 & -9.90 & 1.65 & -5.28 & 0 & -4.13 & -0.66 & -3.60 \\
\hline
\end{tabular}

\section{EDDY-CURRENT LOSSES IN SOLID BACK-IRON}

\section{A. Calculation method}

Lawrenson et al [11] (see also [12]) derived expressions to calculate the eddy-current losses in solid iron poles based on two-dimensional field calculations. They use a three layer model:

1. a layer with perfect iron (resistivity and permeability both infinite)

2. a layer of air (the air gap)

3. a layer of solid iron with a realistic resistivity and permeability.

On the boundary between layers 1 and 2, there is a surface current density, resulting in a traveling wave of magnetic flux density in the air gap. The eddy-currents in the third layer are modeled and the losses in this layer are calculated. End effects are neglected. The effect of the eddy-currents on the magnetic field is included.

The eddy-current losses per square meter of surface area $P_{A}$ are given by

$P_{A}=\frac{\hat{B}_{0}^{2} v^{2}}{4 \rho_{F e} \operatorname{Re} \gamma}$

where

$\hat{B}_{0}$ is the amplitude of the flux density wave

$v$ is the speed of the flux density wave

$\rho_{F e}$ is the resistivity of iron

$\gamma$ can be written as

$\gamma^{2}=\frac{4 \pi^{2}}{\lambda^{2}}-j \frac{2}{\delta^{2}}$

where

$\lambda$ is the wave length of the space harmonic,

$\delta$ is the skin depth, given by

$\delta=\sqrt{\frac{2 \rho_{F e}}{\mu_{0} \mu_{r F e} \omega}}$

where the angular frequency $\omega$ is given by

$\omega=\frac{2 \pi v}{\lambda}$

For the space harmonics we are dealing with, the skin depth is much smaller than the wave length. Therefore, $\gamma^{2} \approx-j \frac{2}{\delta^{2}}$

so that

$\operatorname{Re} \gamma \approx \operatorname{Re} \frac{1-j}{\delta}=\frac{1}{\delta}$

Using this, (20) can be written as

$P_{A}=\frac{\hat{B}_{0}^{2} v^{2} \delta}{4 \rho_{F e}}$

\section{B. Results}

In the calculations, it is assumed that

the relative magnetic permeability of the solid back-iron is $\mu_{r F e}=200$,

- the resistivity of the solid back-iron is $\rho_{F e}=0.2 \mu \Omega \mathrm{m}$.

This equation has been used to calculate the eddy-current losses in the back-iron of the two generators. The results are given in Table IV for the space harmonics until the eleventh. For higher space harmonics, the losses are small and the validity of the models is questionable because the assumption that the field lines cross the air gap perpendicular may be reasonable for space harmonics with a wave length larger than or equal to the slot pitch, but not for the higher space harmonics with a much smaller wave length.

From these calculations, it appears that the eddy-current losses in the solid back-iron of a generator with a full-pitch winding (I) are negligible, as already indicated in [9].

Further, it appears that the eddy-current losses in the backiron of machines with fractional pitch windings where the number of coils is equal to the number of teeth (II-VII) are much larger, mainly because of the sub-harmonics. The combination of 3 teeth with 3 coils per 2 poles has low losses, but this combination is not so interesting because of the poor winding factor. The combination of 9 teeth with 9 coils per 8 poles may be interesting because the losses may be acceptable, while the winding factor for this machine rather high.

It also appears that the eddy-current losses in the back-iron of machines with fractional pitch windings with a coil around every second tooth (VIII-XI) are again much larger. These losses are unacceptably high and lead to huge cooling problems in the translator.

TABLE IV

LOSSES CAUSED BY THE SPACE HARMONICS (W)

\begin{tabular}{|r|r|r|r|r|r|r|r|r|r|r|}
\hline & 1 & 2 & 4 & 5 & 7 & 8 & 10 & 11 & total \\
\hline $\mathrm{I}$ & 0 & 0 & 0 & 8 & 2 & 0 & 0 & 1 & 11 \\
\hline $\mathrm{II}$ & 0 & 85 & 5 & 6 & 2 & 2 & 1 & 1 & 102 \\
\hline $\mathrm{III}$ & 1369 & 0 & 15 & 2 & 3 & 1 & 1 & 1 & 1392 \\
\hline $\mathrm{IV}$ & 104 & 98 & 0 & 211 & 0 & 0 & 0 & 0 & 415 \\
\hline $\mathrm{V}$ & 296 & 35 & 516 & 0 & 2 & 0 & 0 & 0 & 849 \\
\hline $\mathrm{VI}$ & 741 & 0 & 0 & 0 & 169 & 0 & 0 & 0 & 911 \\
\hline $\mathrm{VII}$ & 741 & 0 & 0 & 651 & 0 & 0 & 0 & 1 & 1393 \\
\hline $\mathrm{VIII}$ & 7301 & 0 & 242 & 12 & 16 & 15 & 18 & 3 & 7606 \\
\hline $\mathrm{IX}$ & 10326 & 5476 & 0 & 86 & 4 & 61 & 9 & 8 & 15969 \\
\hline $\mathrm{X}$ & 11067 & 0 & 0 & 0 & 182 & 0 & 0 & 1 & 11249 \\
\hline $\mathrm{XI}$ & 11067 & 0 & 0 & 698 & 0 & 0 & 0 & 4 & 11769 \\
\hline
\end{tabular}




\section{EXPERIMENTAL MODEL VALIDATION}

An experiment was done to validate the model. The experimental setup consists of a linear PM machine with a fractional pitch winding with 3 teeth with 3 coils per 4 poles. The coils are kept in a fixed position with respect to the magnets and the back iron. The stator winding is connected to an $\mathrm{AC}$ voltage with a varying amplitude and frequency, which results in a pulsating field in the back-iron. The voltage, the current and the power are measured. From the measured power and current, a resistance can be calculated, which includes the losses due to eddy currents.

Fig. 7 depicts the measured and the calculated resistance including the effect of eddy currents in the solid back-iron. The magnets between the coils and the back-iron were removed to eliminate eddy-currents in magnets [13]. According to [9], the eddy-current losses in magnets are much smaller than eddy-current losses in the back-iron at frequencies below $100 \mathrm{~Hz}$.

Already at frequencies around $100 \mathrm{~Hz}$, the measured losses are roughly double the losses at DC, which means that at 100 $\mathrm{Hz}$, the losses in the solid back-iron have the same order of magnitude as the copper losses in the coils.

The model derived above and used to calculate the eddycurrent losses in the solid back-iron calculates the eddy current losses as proportional to the frequency to the power of 1.5. The increase of the losses in the measurements is lower. It seems that at low frequencies, the losses due to eddy-currents are underestimated, while at high frequencies, they are overestimated.

Reasons for the differences between calculations and measurements may be the following.

- Besides eddy-current losses, there may also be hysteresis losses, which have been neglected.

- A linear model with a constant magnetic permeability of iron has been used. In reality, saturation effects may play a role as indicated in [12].

- End effects have been neglected, while the measurement setup was rather small, so that end effects are not negligible.

\section{CONCLUSIONS}

A model for the eddy-current losses in the solid back-iron of PM machines has been presented. There is a reasonable correlation between the trends of the calculated and the measured losses, but the differences in the details are not negligible. The eddy-current losses in machines with distributed full-pitch windings are negligible. In machines with fractional-pitch windings, these eddy-current losses are considerable and depend strongly on the combination of number of teeth and number of poles. In fractional pitch machines with a number of coils of half the number of teeth, these losses are excessive.

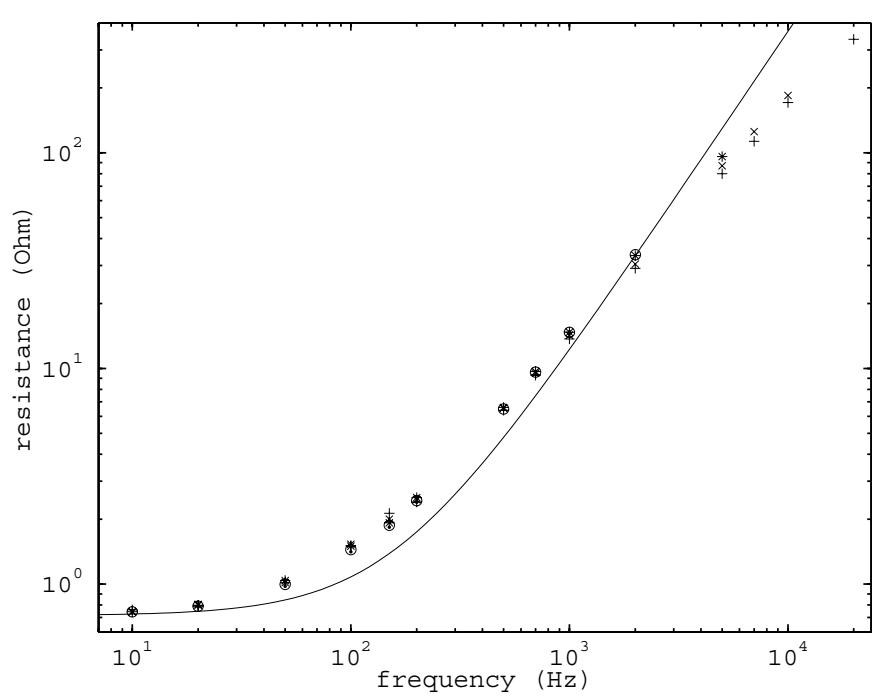

Fig. 7: Measured $(*,+)$ and calculated (-) resistance as a function of frequency for solid back-iron without magnets.

\section{REFERENCES}

[1] H. Polinder, M.E.C. Damen, F. Gardner, "Linear PM generator system for wave energy conversion in the AWS," IEEE Transactions on Energy Conversion, vol. 19, pp. 583-589 (2004).

[2] H. Polinder, M.E.C. Damen, F. Gardner, "Design, modelling and test results of the AWS PM linear generator," European Transactions on Electrical Power, vol. 15, pp. 245-256 (2005).

[3] M. Goden de Sousa Prado, F. Gardner, M.E.C. Damen, H. Polinder, "Modelling and Test Results of the Archimedes Wave Swing," Proceedings of the institution of mechanical engineers Part A, Journal of Power and Energy, vol. 220, pp. 855-868 (2006).

[4] J. Cros, P. Viarouge, "Synthesis of high performance pm motors with concentrated windings", IEEE Transactions on Energy Conversion, vol. 17, pp. 248-253 (2002).

[5] F. Libert, J. Soulard. "Investigation on pole-slot combinations for permanent-magnet machines with concentrated windings", Proc. of the International Conference on Electrical Machines, Cracow, 2004.

[6] R. Wrobel, P.H. Mellor, "Design considerations of a direct drive brushless PM machine with concentrated windings," in Proc. of the 2005 IEEE International conference on electric machines and drives, 2005, pp. 655-658.

[7] A.M. El-Refaie, T.M. Jahns, D.W. Novotny, "Analysis of surface permanent magnet machines with fractional-slot concentrated windings," IEEE Transactions on Energy Conversion, vol. 21, pp. 34-43 (2006).

[8] F. Magnussen, C. Sadarangani, "Winding factors and Joule losses of permanent magnet machines with concentrated windings," in Proc. of the 2003 IEEE International Electric Machines and Drives Conference, 2003, pp. 333 - 339, vol.1.

[9] H. Polinder, M.J. Hoeijmakers, M. Scuotto, "Eddy-current losses in the solid back-iron of permanent-magnet machines with concentrated fractional pitch windings," in Proc. of the 2006 IEE International Conference on Power Electronics, Machines and Drives, Dublin, 4-6 April 2006, pp. 479-483.

[10] H. Polinder, J.G. Slootweg, M.J. Hoeijmakers, J.C. Compter. "Modelling a linear PM machine including magnetic saturation and end effects: maximum force to current ratio", IEEE Transactions on Industry Applications, vol. 39, pp. 1681-1688 (2003).

[11] P.J. Lawrenson, P. Reece, M.C. Ralph. "Tooth-ripple losses in solid poles", Proceedings of the IEE, vol. 113, pp. 657-662 (1966).

[12] K. Oberretl. "Eddy current losses in solid pole shoes of synchronous machines at no-load and on load", IEEE Transactions on Power Apparatus and Systems, vol. 91 pp. 152-160 (1972).

[13] H. Polinder, M.J. Hoeijmakers. "Eddy-current losses in the segmented surface-mounted magnets of a PM machine", IEE Proceedings - Electric Power Applications, vol. 146, pp. 261-266 (1999). 